\title{
Ambiguity and Anaphora with Plurals in Discourse
}

\author{
Nicholas Asher \& Linton Wang \\ University of Texas at Austin
}

\begin{abstract}
We provide examples of plurals related to ambiguity and anaphora that pose problems or are counterexamples for current approaches to plurals. We then propose a dynamic semantics based on an extension of dynamic predicate logic $\left(\mathrm{DPL}^{+}\right)$to handle these examples. On our theory, different readings of sentences or discourses containing plurals don't arise from a postulated ambiguity of plural terms or predicates applying to plural DPs, but follow rather from different types of dynamic transitions that manipulate inputs and outputs from formulas or discourse constituents. Many aspects of meaning can affect the type dynamic transitions: the lexical semantics of predicates to the left and right of a transition, and number features of DPs and discourse constraints like parallelism.
\end{abstract}

\section{Introduction and Motivating Data}

Sentences containing noun phrases often have several different readings-e.g.:

(1) a. Three students wrote a paper.

(Krifka 1996)

b. Three boys invited four girls to the party.

Sentence (1a) has two familiar readings: a distributive and a collective reading. The distributive reading of (1a), implies for each of the three students introduced that they each wrote a paper. The collective reading implies that the three students introduced wrote a paper together. Sentence (1b) has another possible reading besides the distributive and collective ones, the cumulative reading: the boys involved in the inviting relation are three and the girls involved in inviting relation are four but the exact distribution of boys to girls is left underspecified in the sentence's logical form. Like most linguists and philosophers who have worked on plurals, however, we take the difference between cumulative, distributive, and collective readings to be an ambiguity that should show up as differences in logical form.

Besides these familiar readings, we note a difference in distributive readings. Consider:

(2) a. All students go to school.

b. Three students each wrote a paper.

In (2a), we have a simple distributive reading: the VP predicate applies on the individual students singly; every student has the property of going to school. (2b) gives rise to a dependent reading: the VP predicate not only applies on student singly in this reading, but there is also a dependency between the objects quantified over by the subject and object noun phrases respectively. (2b) sets up a dependency between each of the students and a paper they wrote. This dependency, which some languages like Hungarian mark grammatically (via determiner reduplication, Farkas 1997), has substantially different discourse effects from simple distributivity, as we'll soon see. 


\section{Previous Approaches to Ambiguity with Plurals}

Current approaches to the different readings of plural expressions like (1a) fall generally into three camps (more distinctions are available in Lasersohn 1995): (a) those claiming that plural nominal terms like three students are ambiguous, (b) those claiming that predicates like write are ambiguous and (c) those claiming plural expressions are unspecified in the sense that different plural readings correspond to different situations that make a sentence containing plurals true. We argue that none of approaches (a)-(c) are right and propose an interpretation of plural readings based on an ambiguity of dynamic transitions. First, we reject approach (c) since we think examples like (1a) generate a real ambiguity at logical form rather than an unspecified meaning. ${ }^{1}$

A problem for approach (a), the term-ambiguity approach (e.g. Scha 1981, Boolos 1984, Gillon 1987, Schein 1993, Krifka 1996, van den Berg 1996, etc.), is that it cannot handle sentences in which both readings for the plural DP seem required. Consider the following two sentences. ${ }^{2}$

(3) a. Three students worked tirelessly and mowed the whole meadow. ${ }^{3}$

b. Three students mowed the whole meadow together. They worked tirelessly.

In (3a), we interpret the plural term three students distributively with respect to worked timelessly and collectively with respect to mowed the whole meadow. But there is no way to access simultaneously the two interpretations on any sensible, compositional analysis of (3a) with a term-ambiguity approach. Example (3b) shows that this problem carries over to the interpretation of anaphoric pronouns. If "three students" is interpreted collectively so as to combine properly with the "mowed the whole meadow, then the pronoun they must refer to the collectively interpreted antecedent; in other words, the reading of the antecedent must "persist" in the interpretation of the pronoun and this results in the wrong reading of the second sentence. ${ }^{4}$ On the other hand, if the term some students in the first sentence of (3b) is ambiguous, then the pronoun should have different possible interpretations. However, this does not seem to be the case. The first sentences of (3b) by themselves don't obey the standard tests for being ambiguous sentences. ${ }^{5}$

Cumulative readings for plural sentences also pose a problem for approach (a). According to a term-ambiguity approach to get the cumulative reading for (1b), we must give a cumulative interpretation for the plural terms "three boys" and "four girls". However, since there are different possible inviting relations between boys and girls, we should be able to get many different cumulative interpretations for "three boys" and "four girls"; these terms should be many ways ambiguous. Once again this doesn't seem to be the case.

There are two solutions to the problem of cumulative interpretation. Scha (1981) proposes that the interpretation for cumulative reading in (1b) is that the boys which invited girls are three and the girls which are invited by boys are four. Schein (1993) and Landman (2000) propose that a cumulative interpretation requires a second order formula saying essentially that there is one large event with a finite number of inviting sub-events whose agents are the three boys and whose patients are the three girls and for each sub-event some subset of the boys invites either distributively or collectively some subset of the girls. These two interpretations sound right to us, but it's completely unclear how this would fit into a term ambiguity approach to plural DPs. ${ }^{6}$ To formulate 
these two approaches properly we need to consider the sentence as a whole and so the ambiguity cannot lie just in the two DPs but in their relation to each other as well as to the predicate. ${ }^{7}$

Approach (b), the predicate-ambiguity approach (e.g. Link 1983, Landman 2000, Winter 2001, etc.), also faces problems. This approach postulates an ambiguity among predicate interpretations; predicates either receive a distributive interpretation with respect to their plural arguments or a collective one (Winter, 2001). While this handles example (3a), we run into problems with cumulative readings like that in (1b) above. If the cumulative reading of (1b) derives solely from the interpretation of the predicate invite, then since we can have a lot of different possible inviting relations between boys and girls, it appears as though the predicate needs to be typed both for individual and set-like arguments. This is either inconsistent (if the two types have no meet) or at least requires an account of how to interpret a predicate with respect to two types simultaneously. We could perhaps avoid this problem by postulating a special type of predicate for cumulative interpretations. But in any case, simply postulating an ambiguity at the level of the predicate won't explain how distributive and collective interpretations affect subsequent sentences in discourse. In (4), the first sentence has both a collective and a distributive reading. But only the distributive interpretation suitably, and dynamically, formalized gives an appropriate antecedent for the pronoun them; somehow the distributive interpretation has to "carry over" to the next sentence. Further evidence for the carry over effect is that if the first sentence is interpreted distributively, this distributive way of reading plurals naturally carries over to the second sentence as well; that is, it is naturally interpreted distributively rather than collectively or cumulatively.

Three students wrote a paper. They sent them to L \& P.

(Krifka 1996)

If plural readings are interpreted as a feature of the predicate alone, it is difficult if not impossible to explain these carry over effects. ${ }^{8}$

The carry over effects of certain readings are quite striking. For example, singular pronouns whose antecedents occur under the scope of some non-existential quantifier appear to demand a particular sort of dynamic transition. Consider the examples in (5):

(5) a. Three students each wrote a paper. They sent it to L\&P. (Krifka 1996)

b. (Students have the option of either taking an exam or write a paper). Most students wrote a paper. They thought that writing it would be easier than studying for the exam.

(A. Gillies, p.c.)

The singular pronoun "it" in $(5 a, b)$ is acceptable to many speakers of English. But it does not function like a regular singular pronoun, whose antecedent would guarantee a single individual as the pronoun's denotation. In dynamic semantic terms, the indefinite NP falls within the scope of a plural quantifier whose distributive reading forced by each and thus entails that there are at least three papers that the it refers to. In the jargon of standard dynamic semantics, the indefinite DP a paper, or rather the discourse referent it introduces, is inaccessible to the pronoun. ${ }^{9}$

In fact $(5 a, b)$ don't have a simple distributive reading but rather a dependent one (for every student there is at least one paper that they wrote). This is crucial to the acceptability of these examples, in which singular pronouns refer to what are, dynamically speaking, inaccessible antecedents. A simple distribution over the subject NP 
fails to make the examples in $(5 a, b)$ any more acceptable. If a simple distributive reading permitted links to variables that are inaccessible, then $(6 a, b)$ should be as good as (5a,b), but they are not.

(6) a. Every boy studied diligently on his own. ??He got an A.

b. Every farmer who bought a donkey brought it home. *He treated it kindly.

The dependent reading also appears to decay as discourse progresses. Consider (7).

(7) Three students each wrote a paper. They sent it to L\&P. ?They had worked very hard on it.

While (5a) is fine, (7) is already degraded; it appears that the dependent reading is short lived discursively.

\section{Our Approach}

We account for our observations in a compositional way by an essential appeal to a dynamic discourse semantics like SDRT (Asher 1993, Asher \& Lascarides 2003). From our discourse perspective, a discourse structure involves discourse constituents with rhetorical relations between them. Each clause gives rise to a constituent, but there are also sub-sentential constituents. Here we abstract away from particular discourse relations to consider only transitions. Transitions (marked by ;) occur not only between clauses or, rather, discourse constituents, but also within a clause-e.g., between a quantifier's restrictor and nuclear scope. The major feature of our approach is to distinguish several types of transitions: $\operatorname{Dis}(x), \operatorname{Dep}(x, y), \operatorname{Col}(x)$ and $\operatorname{Cum}(x)$ are different sorts of transitions that determine distributive, dependent, collective and cumulative readings respectively.

Although the simple distributive transition is the default, plural DPs give rise to other possible transitions. Some plural DPs are inherently distributive (e.g., each); others are more catholic. Similarly, some predicates have selectional restrictions that require their arguments to be individuals (the inherently distributive predicates); some require their arguments to be plural or group like (the collective predicates) and some are indifferent; the picky predicates will only work if their inputs are conditioned by certain transitions. For pronouns, singular pronouns demand a single value for a given dynamic input, while plural pronouns are less picky and don't demand any cardinality constraints on the number of objects associated with a variable in a given information state; thus singular pronouns are only felicitous in very special environments. To account for this, we'll take all number marking on DPs to be semantically significant.

Our semantics uses a simple first order model and structured sets of assignments as the inputs and outputs of the interpretation of logical forms. We thus avoid any appeal to sums of individuals or complex eventualities to explain plurality (cf. Krifka 1996). To simplify our semantics, we'll separate out the notion of a transition (;) and conditions on those transitions by expressing the latter with transition formulas. For instance, the transition formula ' $j^{D i s}(x)$ ' (for distributive "jump" on $x$ ) must pass each subset of the input assignments with a single value of $x$ "one at a time" to the interpretation of the formula on its right. The transition formula $j^{D e p}(x, y)$ must pass each 
subset of the input assignments with a single value of $x$ "one at a time" to the interpretation of the formula on its right while collecting sets of values of $y$ for each $x$ to pass onto the formula on its right. Once the interpretation of the input is set, it will be passed onto other constituents across other dynamic transitions unless it is reset either by contextual, pragmatic or semantic features. This explains the defeasible carry over effect noted earlier. To illustrate consider again (3a). The dynamic logical form for the first sentence has a transition between the nuclear scope of the quantifier three students and the restrictor. This transition is ambiguous between collective and distributive readings. In the logical form $\left(3 a^{\prime}\right)$ for $(3 a)$, the lexical semantics of the verbs in the coordinate VP force the transitions to their left to be distributive and collective respectively; the transition marked with $\operatorname{Col}(x)$ requires that the values of all the assignments for $x$ in the input set be considered all at once in the interpretation of the formula to the right of the transition. $\left(3 \mathrm{a}^{\prime}\right)$ exemplifies how to reset an input structured set of assignments.

$\left(3 \mathrm{a}^{\prime}\right)$ three students $(x) ; j^{D i s}(x)$; worked tirelessly $(x) ; j^{C o l}(x) ; \operatorname{mowed}(x)$

With our different types of transitions, we can easily represent different readings of plural sentences without a complex semantics for DPs or predicates. Below are our representations for the different readings of $(1 \mathrm{~b})$ :

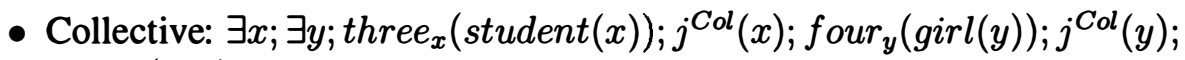
invite $(x, y)$

- Cumulative: $\exists x ; \exists y ;$ three $_{x}(\operatorname{student}(x)) ; j^{C u m}(x) ;$ four $_{y}(\operatorname{girl}(y)) ; j^{C u m}(y)$; invite $(x, y)$

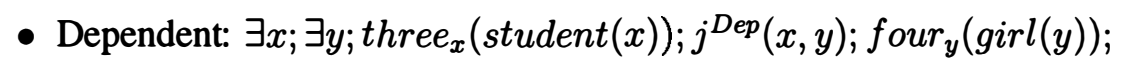
invite $(x, y)$

We now turn to details. First, we specify the lexical semantics of determiners and predicates. All determiners and predicates have univocal lexical entries, as far as the distinction between different plural readings is concerned. Some predicates will select for collective or plural type arguments and others will not, but these lexical entries will help resolve ambiguity rather than cause it. The ambiguity of plural sentences is not a lexical matter for us. All determiners introduce a presupposition that there is a set satisfying the restrictor, an assertion that may involve either a first order or non first order quantifier, or a first order definable "counting" predicate on the presupposed set (e.g. a, the, one, two, three, more than $n) .{ }^{10}$ So for instance (8a) will introduce the logical form in (8b)
(8) a. three boys
b. $\lambda Q\left(\right.$ three $\left._{x}(\operatorname{boy}(x)) ; Q(x)\right)$

We will assume that such logical forms receive existential closure at the level of each constituent, as in DRT.

All determiners in our treatment license witnesses whether as part of their assertive content or their presuppositions. The function of determiners is to define what sorts of witnesses are made available for reference in subsequent discourse. Thus natural language determiners for us play a different role from the quantifiers $\exists$ and $\forall$ in DPL 
(Groenendijk \& Stokhof 1991), or first order logic. In standard dynamic semantics, e.g. DPL, only indefinites license witnesses. In particular, DPL's treatment of all determiners that are downward monotonic in their first argument poses a problem for us. For instance, the universal quantifier $\forall$ translates to $\neg \exists \neg$ which is a test on inputs and cannot provide any useful witness for the variable governed by the quantifier or any other variable within its scope; its semantic definition entails that its output is identical to its input when the input passes the test (and it outputs nothing otherwise). This furnishes the core of DPL's explanation of why $(6 a, b)$ are bad. However, for the same reason, DPL's treatment of universal quantification also blocks any kind of witness information for the plural pronoun. So simple plural anaphora like that in (9) fails:

(9) Every farmer who bought a donkey brought it home with him. They used them to work in the fields.

We don't propose to give up on the generally accepted truth conditions for every each and most. In order to supply witness sets for these sorts of quantified DPs, we instead look to their presuppositions and how they interact with the asserted content. The presupposition of every farmer, most farmers, etc. is that there is a set of farmers. When these presuppositions interact dynamically with the asserted content of these DPs they produce side effects on updates that provide the appropriate sorts of witnesses for plural anaphora. These side effects are for us part of the content of a determiner.

To understand these side effects a bit better, we need to digress for a minute on treatments of presupposition in a discourse semantics. Extending van der Sandt (1992), SDRT countenances presupposed information as a separate constituent to be added to the discourse context. Because of the way SDRT updates discourse content with conditions that state rhetorical relations, SDRT has a particular background relation in which the background information comes first and checks the input to see whether the presupposition is satisfied. In this paper we've abstracted away from discourse relations, but the satisfaction of the presupposition and assertion still follow the general pattern. The point is that when the presupposition's content is updated with the assertion's content, the information associated with the variables introduced by the presupposition changes.

To make this a bit more concrete, let's write down the lexical entries for determiners like every, each or all. All of these determiners have similar presupposed and asserted contents: roughly, if we lambda bind variables for the restrictor $(P)$ and for the nuclear scope $(Q)$, the presupposition is that there is a set of all Ps and the assertion is that everything that is a $P$ is a $Q$. Now we have to take account of how the presupposition and assertion interact. Let's assume that the presupposition attaches to the asserted component. What happens after updating an information state with the presupposed and asserted information? The assertion entails that every element in the presupposed set now also satisfies the nuclear scope of the quantifier. And it is this that we pick up anaphorically when we refer with a plural pronoun. In particular, this means that in (9) all the farmers who bought donkeys and brought them home with them have the property described in the second sentence.

Because of the dependency information of the nuclear scope on the restrictor set, we can also get other sets using our transition operators; in other words, they affect the output from the update. Different determiners may have different transitions; for 
example every and each will allow for both distributive and dependent transitions in the side effect conditions. We'll include these side effect conditions in the lexical entry for the determiner. As is usual in SDRT, we'll assume that presuppositions attach to asserted content via a Background relation. ${ }^{11}$ We can prove semantically that any update that satisfies the presupposition and assertion will affect the value of the variable $x$ that serves as the witness set for the quantifier, but we need to stipulate the particular distributional effects that each determiner has and we also have to stipulate that $x$ is assigned the set of all objects that satisfy the presupposition and assertion. So we'll put these side effect conditions as part of the lexical entry of the determiner and part of logical form. For example here is the entry for every with a dependent reading; note that the dependent variable is itself underspecified with a '?' (and will only be filled in during the compositional process):

$\lambda P \lambda Q[$

- $\pi_{1}: \exists x ; P(x)$;

- $\pi_{2}: \neg \exists y ;(P y ; \neg Q y)$

- $\left.\operatorname{Background}\left(\pi_{1}, \pi_{2}\right) ; j^{D e p}(x, ?) ; \operatorname{all}_{x}(P(x)) ; Q(x)\right]$

The entry for most, like the entry above, makes use of the counting predicate all to ensure the maximality of the set assigned to $x$; the side effect conditions also encode a dependency transition and ensure that the set picked out by the presupposition also satisfies the property specified by the nuclear scope of the quantifier. But the entry makes use of a non first order definable quantifier MOST with the standard interpretation (a fresh variable $y$ is used in order to avoid any clash with value assigned to $x$ ).

$\lambda P \lambda Q[$

- $\pi_{1}: \exists x ; P(x)$

- $\pi_{2}: \operatorname{MOST}_{y}(P y, Q y)$

- Background $\left.\left(\pi_{1}, \pi_{2}\right) ; j^{D e p}(x, ?) ; \operatorname{all}_{x}(P(x)) ; Q(x)\right]$

These side effect conditions allow us to get the intended, dependent readings of the plural pronouns in the second sentence of (9); in particular we get that the farmers who bought donkeys and brought them home use the donkeys they bought and brought home.

\section{Augmented Dynamic Predicate Logic (DPL $\left.{ }^{+}\right)$}

We turn now to the formal underpinnings of our approach, $\mathrm{DPL}^{+}$, which is an extension of DPL. ${ }^{12}$. We expand the DPL language to include information about different sorts of transitions; to reduce typographical clutter, we'll keep the DPL transition ';' and add our subscripts as formulas in their own right. Second we revise what are the inputs and outputs to formulas in the semantics; instead of individual assignments, structured sets of assignments of individuals to variables will serve as the inputs and outputs. 


\section{Definition 1 Language of DPL ${ }^{+}$}

1. Logical Symbols

The usual DPL logical symbols—including variables, connectives $(\neg, ;)$ and quantifier $(\exists)$.

2. Non-Logical Symbols

(a) The Usual Predicate Symbols

(b) Transition Predicates: $j^{D e p}$ (of arity 2 ), $j^{C o l}, j^{C u m}, j^{D i s}$ (each of arity 1 )

(c) Counting Predicates: all, some, a, one, two, ..., at_least_n, at_most_n, ...

(d) Non First Order Definable Quantifiers: $M A N Y, M O S T$, etc.

\section{Definition 2 Syntax of DPL ${ }^{+}$}

1. $P\left(t_{1}, \ldots, t_{n}\right)$ is an atomic formula, in which $t_{i}$ is a term and $P$ an n-ary predicate.

2. the usual DPL clauses for formulas involving;, $\neg$, and $\exists$.

3. For any non first order definable quantifiers $D, D_{t_{i}}(\varphi, \psi)$ is a formula if $\varphi$ and $\psi$ are formulas and $t_{i}$ is an variable.

4. For any counting predicates $D$ like all, $n$, the, $a$, and some, $D_{t_{i}}(\varphi)$ is a formula if $\varphi$ is a formula and $t_{i}$ is an variable.

The syntax of $\mathrm{DPL}^{+}$is similar to DPL except for the formulas representing information about transitions, the counting predicate formulas, and the non-first order definable determiners.

A model for the language of $\mathrm{DPL}^{+}$is defined by a pair, $M=<D, I>$, where $D$ is a non-empty set (of individuals) and $I$ is an interpretation function. For a constant $c_{j}, I\left(c_{j}\right) \in D$. For a n-place predicate $P^{n}$, we define $I\left(P^{n}\right) \subseteq(D \cup \wp(D))^{n}$ instead of defining $I\left(P^{n}\right) \subseteq D^{n}$ in the usual way, in order to account for collective and cumulative readings, in which predications occur on sets of entities. A function $g$ is an assignment function iff $g:(V \cup C) \rightarrow D$, where $V$ is the set of variables and $C$ is the set of constants, and $g\left(c_{i}\right)=I\left(c_{i}\right) . S_{F}$ is an information state iff $S_{F}=\left\{<g, f_{g}\right\rangle \mid g \in S$ and $\left.S \subseteq \$\right\}$ in which $\$$ is the set of assignment functions, and $f_{g}: V \rightarrow(\$ \cup \wp(\$)) \times(D \cup \wp(D))$-where if $f_{g}\left(t_{i}\right)=(A, B)$, then $g \in A$ is a function that records dependency and assignment information on assignments. We will make use of this function in defining the semantics of transition operations. Finally, the projection function $\mu^{1,2}$ yields either the first or the second element of the pairs that make up $f_{g}\left(t_{i}\right) \cdot \mu^{1}\left(f_{g}\left(t_{i}\right)\right)$ partitions the set of assignments according to how many objects are assigned to $x$ by assignments in a given information state, while $\mu^{2}\left(f_{g}\left(t_{i}\right)\right)$ is the actual assignment to $x$ by the pair $\left\langle g, f_{g}\right\rangle$ in the information state.

The collection function $\delta$ collects up values assigned to a variable $t_{i}$ by an element in the partition on assignments given by $\left.\mu^{1}\left(f_{g_{j}}\left(t_{i}\right)\right)\right)$ and is defined as follows: $\delta\left(\mu^{1}\left(f_{g_{j}}\left(t_{i}\right)\right)\right)=\left\{g_{h}\left(t_{i}\right) \mid g_{h} \in \mu^{1}\left(f_{g_{j}}\left(t_{i}\right)\right)\right\}$ if $\left.\left.\mu^{1}\left(f_{g_{j}}\left(t_{i}\right)\right)\right) \neq g_{j}\left(t_{i}\right) ; \mu^{1}\left(f_{g_{j}}\left(t_{i}\right)\right)\right)=$ $g_{j}\left(t_{i}\right)$, otherwise. The interpretation of formulas is a function [.] $]_{M}: \varphi \rightarrow \$_{F} \times \$_{F}$ that $\$_{F}$ is the set of information states. For any information state $S_{F}, S=\left\{g \mid<g, f_{g}>\epsilon\right.$ $\left.S_{F}\right\}$ and $F=\left\{f_{g} \mid<g, f_{g}>\in S_{F}\right\}$. In definition 3, we give the semantics for the 
transition or jump formulas. The initial input information state $S_{F}$ must satisfy the following two constraints: (i) for any $g_{j}$ and $t_{i}, \mu^{1}\left(f_{g_{j}}\left(t_{i}\right)\right)=S$, and (ii) for any $g_{j}$ and $t_{i}, \mu^{2}\left(f_{g_{j}}\left(t_{i}\right)\right)=g_{j}\left(t_{i}\right)$.

Below we give the semantics of the atomic formulas, which for us includes not only standard DPL formulas but those transition formulas that have an effect on how assignments are structured and what variables in fact get assigned in information states.

Definition 3 Semantics for Atomic Formulas of DPL ${ }^{+}$

1. $S_{F}\left[P\left(t_{1}, t_{2}, \ldots, t_{n}\right)\right]_{M} S_{F}^{\prime}:=S_{F}=S_{F}^{\prime}$, and for any $g_{j} \in S^{\prime}$, $<\mu^{2}\left(f_{g_{j}}^{\prime}\left(t_{1}\right)\right), \ldots, \mu_{2}^{2}\left(f_{g_{j}}^{\prime}\left(t_{n}\right)\right)>\in P^{M}$.

2. $S_{F}\left[j^{D e p}\left(t_{i}, t_{h}\right)\right]_{M} S_{F^{\prime}}^{\prime}:=$

(a) $S=S^{\prime}$

(b) for any $g_{j} \in S^{\prime}$ and $t_{k}, \mu^{2}\left(f_{g_{j}}^{\prime}\left(t_{k}\right)\right)=\mu^{2}\left(f_{g_{j}}\left(t_{k}\right)\right)$,

(c) for any $g_{j} \in S^{\prime}, \mu^{1}\left(f_{g_{j}}^{\prime}\left(t_{h}\right)\right)=\left\{g_{m} \mid g_{m}\left(t_{i}\right)=g_{j}\left(t_{i}\right)\right.$ and $g_{m} \in$ $\left.\mu^{1}\left(f_{g_{j}}\left(t_{h}\right)\right)\right\}$, and for any $t_{k}$ such that $t_{k} \neq t_{h}, \mu^{1}\left(f_{g_{j}}^{\prime}\left(t_{k}\right)\right)=\mu^{1}\left(f_{g_{j}}\left(t_{k}\right)\right)$.

3. $S_{F}\left[j^{C o l}\left(t_{i}\right)\right]_{M} S_{F}^{\prime}:=$

(a) $S=S^{\prime}$,

(b) for any $g_{j} \in S^{\prime}, \mu^{2}\left(f_{g_{j}}^{\prime}\left(t_{i}\right)\right)=\delta\left(\mu^{1}\left(f_{g_{j}}\left(t_{i}\right)\right)\right)$, and for any $t_{k}$ such that $t_{k} \neq t_{i}, \mu^{2}\left(f_{g_{j}}^{\prime}\left(t_{k}\right)\right)=\mu^{2}\left(f_{g_{j}}\left(t_{k}\right)\right)$,

(c) for any $g_{j} \in S^{\prime}$ and $t_{k}, \mu^{1}\left(f_{g_{j}}^{\prime}\left(t_{k}\right)\right)=\mu^{1}\left(f_{g_{j}}\left(t_{k}\right)\right)$.

4. $S_{F}\left[j^{C u m}\left(t_{i}\right)\right]_{M} S_{F}^{\prime}:=$

(a) $S=S^{\prime}$,

(b) for any $g_{j} \in S^{\prime}, \mu^{2}\left(f_{g_{j}}^{\prime}\left(t_{i}\right)\right) \subseteq \delta\left(\mu^{1}\left(f_{g_{j}}\left(t_{i}\right)\right)\right)$ and $\cup\left\{\mu^{2}\left(f_{g_{h}}^{\prime}\left(t_{i}\right)\right) \mid g_{h} \in\left(\mu^{1}\left(f_{g_{j}}^{\prime}\left(g_{j}, t_{i}\right)\right)\right)\right\}=\delta\left(\mu^{1}\left(f_{g_{j}}^{\prime}\left(, t_{i}\right)\right)\right)$, and for any $t_{k}$ such that $t_{k} \neq t_{i}, \mu^{2}\left(f_{g_{j}}^{\prime}\left(t_{k}\right)\right)=\mu^{2}\left(f_{g_{j}}\left(t_{k}\right)\right)$,

(c) for any $g_{j} \in S^{\prime}$ and $t_{k}, \mu^{1}\left(f_{g_{j}}^{\prime}\left(g_{j}, t_{k}\right)\right)=\mu^{1}\left(f_{g_{j}}\left(g_{j}, t_{k}\right)\right)$.

5. $S_{F}\left[j^{D i s}\left(t_{i}\right)\right]_{M} S_{F}^{\prime}:=$

(a) $S=S^{\prime}$,

(b) for any $g_{j} \in S^{\prime}, \mu^{2}\left(f_{g_{j}}^{\prime}\left(t_{i}\right)\right)=g_{j}\left(t_{i}\right)$, and for any $t_{k} \neq t_{i}$, $\mu^{2}\left(f_{g_{j}}^{\prime}\left(t_{k}\right)\right)=\mu^{2}\left(f_{g_{j}}\left(t_{k}\right)\right)$,

(c) for any $g_{j} \in S^{\prime}, \mu^{1}\left(f_{g_{j}}^{\prime}\left(t_{i}\right)\right)=S^{\prime}$, and for any $t_{k} \neq t_{i}$, $\mu^{1}\left(f_{g_{j}}^{\prime}\left(t_{k}\right)\right)=\mu^{1}\left(f_{g_{j}}\left(t_{k}\right)\right)$.

6. $S_{F}\left[\exists t_{i}\right]_{M} S_{F}^{\prime}:=$

(a) $S={ }_{t_{i}} S^{\prime}$, i.e. for any $g_{j} \in S$, there is an $g_{k}^{\prime} \in S^{\prime}$ that $g_{j}={ }_{t_{i}} g_{k}^{\prime}$ and for any $g_{k}^{\prime} \in S^{\prime}$, there is $g_{j} \in S$ that $g_{j}={ }_{t_{i}} g_{k}^{\prime}$, 
(b) for any $g_{j} \in S^{\prime}, \mu^{2}\left(f_{g_{j}}^{\prime}\left(t_{i}\right)\right)=g_{j}\left(t_{i}\right)$, and for any $t_{k}$ such that $t_{k} \neq t_{i}$, $\mu^{2}\left(f_{g_{j}}^{\prime}\left(t_{k}\right)\right)=\mu^{2}\left(f_{g_{j}}\left(t_{k}\right)\right)$,

(c) for any $g_{j}$ and $t_{k}, \mu^{1}\left(f_{g_{j}}^{\prime}\left(t_{k}\right)\right)=\left\{g_{h} \in S^{\prime} \mid\right.$ there is an $g_{k} \in \mu^{1}\left(f_{g_{f}}\left(t_{i}\right)\right)$ that $g_{f}={ }_{t_{i}} g_{j}$ and $\left.g_{h}={ }_{t_{i}} g_{k}\right\}$.

Notice that transition formulas, though atomic, are not simple tests, but transform the structure of the output assignments.

We now tackle the recursive rules for complex formulas. For lack of space we won't give the rules for the non first order definable quantifiers $M O S T, M A N Y$, etc. but they are what one would expect dynamically (cf. van den Berg 1996) and don't affect anaphora (they will just define tests). But we will detail the semantics of the counting formulas, in particular the predicate $a_{l} l_{x}$, as these are special to our approach and affect anaphora.

Definition 4 Semantics for Complex Formulas of DPL ${ }^{+}$

1. $S_{F}[\varphi ; \psi]_{M} S_{F}^{\prime}:=\exists S_{F^{\prime \prime}}, S_{F}[\varphi]_{M} S_{F}^{\prime \prime}$ and $S_{F}^{\prime \prime}[\psi]_{M} S_{F}^{\prime}$.

2. $S_{F}[\neg \varphi]_{M} S_{F}^{\prime}:=S=S_{F^{\prime}}, \neg \exists S_{F^{\prime \prime}}, S_{F}[\varphi]_{M} S_{F}^{\prime \prime}$

3. $S_{F}\left[\text { some }_{t_{i}}(\varphi)\right]_{M} S_{F}^{\prime}:=S_{F}[\varphi]_{M} S_{F}^{\prime}$, and for any $g_{j} \in S^{\prime}, \mid \delta\left(\mu^{1}\left(f_{g_{j}}^{\prime}\left(t_{i}\right)\right) \mid \geq 1\right.$.

4. $S_{F}\left[a_{t_{i}}(\varphi)\right]_{M} S_{F}^{\prime}:=S_{F}[\varphi]_{M} S_{F}^{\prime}$, and for any $g_{j} \in S^{\prime}, \mid \delta\left(\mu^{1}\left(f_{g_{j}}^{\prime}\left(t_{i}\right)\right) \mid=1\right.$.

5. $S_{F}\left[n_{t_{i}}(\varphi)\right]_{M} S_{F}^{\prime}:=S_{F}[\varphi]_{M} S_{F}^{\prime}$, and for any $g_{j} \in S^{\prime}, \mid \delta\left(\mu^{1}\left(f_{g_{j}}^{\prime}\left(t_{i}\right)\right) \mid=n\right.$.

6. $S_{F}\left[\operatorname{all}_{t_{i}}(\varphi)\right]_{M} S_{F}^{\prime}:=S_{F}[\varphi]_{M} S_{F}^{\prime}$, and for any $g_{j} \in S^{\prime}, \delta\left(\mu^{1}\left(f_{g_{j}}^{\prime}\left(t_{i}\right)\right)\right)=\varphi^{M}\left(t_{i}\right)$, in which $\varphi^{M}\left(t_{i}\right)=\left\{g_{i}\left(t_{i}\right) \mid g_{i} \in S\right.$ and $\left.S_{F} \in\left\{S_{F}^{\prime} \mid \exists S_{F}, S_{F}[\varphi]_{M} S_{F}^{\prime}\right\}\right\}$.

7. $S_{F}\left[\text { more_than_n } n_{t_{i}}(\varphi)\right]_{M} S_{F}^{\prime}:=S_{F}[\varphi]_{M} S_{F}^{\prime}$, and for any $g_{j} \in S^{\prime}$, $\left|\delta\left(\mu^{1}\left(f_{g_{j}}^{\prime}\left(t_{i}\right)\right)\right)\right|>n$.

8. $S_{F}\left[\text { at_least_n } n_{t_{i}}(\varphi)\right]_{M} S_{F}^{\prime}:=S_{F}[\varphi]_{M} S_{F}^{\prime}$, and for any $g_{j} \in S^{\prime},\left|\delta\left(\mu^{1}\left(f_{g_{j}}^{\prime}\left(t_{i}\right)\right)\right)\right| \geq$ $n$.

9. $S_{F}\left[\text { exactly_n } n_{t_{i}}(\varphi)\right]_{M} S_{F}^{\prime}:=S_{F}[\varphi]_{M} S_{F}^{\prime}$, for any $g_{j} \in S^{\prime},\left|\delta\left(\mu^{1}\left(f_{g_{j}}^{\prime}\left(t_{i}\right)\right)\right)\right|=n$, and $S_{F}\left[\neg\left(\exists t_{i} ; \text { more_than_ } n_{t_{i}}(\varphi)\right)\right]_{M} S_{F}$.

10. $S_{F}\left[\text { the }_{t_{i}}(\varphi)\right]_{M} S_{F}^{\prime}:=S_{F}\left[\operatorname{all}_{t_{i}}(\varphi)\right]_{M} S_{F}^{\prime}$, and for any $g_{j} \in S^{\prime}, \mid \delta\left(\mu^{1}\left(f_{g_{j}}^{\prime}\left(t_{i}\right)\right) \mid=1\right.$.

The definition 5 defines the semantic number agreement of pronouns and impose number requirements on the values of variables. singular $\left(t_{i}\right)$ represents the number requirements for a singular pronoun. This predicate resembles a counting predicate, except that it do not take any formula in their scope.

Definition 5 Semantics of Number Features of Pronouns

$S_{F}\left[\operatorname{singular}\left(t_{i}\right)\right]_{M} S_{F}^{\prime}:=S_{F}=S_{F}^{\prime}$ and $S_{F}^{\prime}\left[a_{t_{i}}(\mathrm{~T})\right]_{M} S_{F}^{\prime}$. 
The semantic interpretation above extends DPL's semantic interpretation with structured assignment sets. The definition of satisfaction, consequence, and validity follow the definition in DPL in Groenendijk \& Stokhof (1991).

The semantic definition for non-monotonic determiners in $\mathrm{DPL}^{+}$differs from that for the monotonic determiners. The counting predicate must take scope not only over the restrictor but over the nuclear scope as well. Consider for instance:

(10) Exactly five students went to school.

which has non-monotonic determiner in the subject position; we need both restrictor and nuclear scope to fall under the scope of the counting predicate. Here is the logical form for (10):

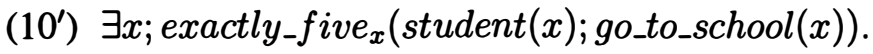

(10) says not that there are exactly five students but that there are exactly five students who went to school. Our definition also works when we have a determiner like exactly_ $n$ in the object DP. Consider the sentence "three boys invited exactly five girls". The simple left-right distributive reading and the dependent reading are given below.

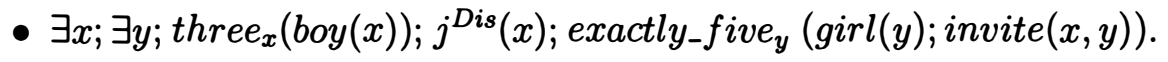

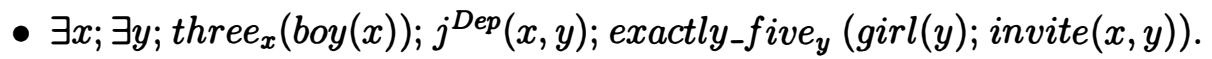

These two logical forms each specify that each of the three boys invited exactly five girls. The second, however, encodes the dependency between each value for $x$ and the five girls invited by that value for $x$. Which five girls are invited depends on the value for $x$. To get the inverse (right left) scope reading, we need to only reverse the dependency, as we see in the formula below.

- $\exists x ; \exists y ; \operatorname{three}_{x}\left(\operatorname{boy}(x) ;\right.$ exactly_five $_{y}\left(\operatorname{girl}(y) ; j^{D e p}(y, x) ;\right.$ invite $\left.\left.(x, y)\right)\right)$.

This formula says that there are exactly five girls such that three boys that invited them; the dependency formula encodes for each girl $y$, the three boys that invited her.

\subsection{Some Examples}

To get a feel for the interpretation of DPL ${ }^{+}$formulas, let's return to (3a) and its logical form $\left(3 a^{\prime}\right)$ and work through how the information states evolve as we interpret each formula in the sequence.

(3a) Three students worked tirelessly and mowed the whole meadow. They had a good time.

$\left(3 \mathrm{a}^{\prime}\right) \exists x ; \operatorname{three}_{x}(\operatorname{student}(x)) ; j^{\operatorname{Dis}}(x) ; \operatorname{work}(x) ; j^{C o l}(x) ; \operatorname{mow}(x) ; j^{D i s}(x)$; good_time $(x)$

(i) At the starting point, we input a structured assignment sets $S_{F}$.

(ii) According to the semantics of ; $S_{F}$ first hits the formula " $\exists x$ " and outputs $S_{F}^{1}$ where $S={ }_{x} S^{1}$, and every member in $F^{1}$ is defined such that the new value for $x$ is encoded as the value of each of the output assignments. Now $S_{F}^{1}$ passes 
through ; to serve as input to the formula "three $($ student $(x))$ ". There is no resetting in "three $(\operatorname{student}(x))$ "; it is just a test. If $S_{F}^{1}$ passes the test, then the output $S_{F}^{2}=$ $S_{F}^{1}$. If $S_{F}^{1}$ fails the test, there is no output from the formula. In the case that $S_{F}^{1}$ passes the test, $S^{1}$ assigns three students in total to the variable $x$ (the first argument of $f_{g}(x)$ for each $g \in S^{1}$ ), though the value of each assignment in $S^{1}$ is just an individual (the second argument of $\left.f_{g}(x)\right)$. Note that there may be other sets of assignments that pass the test "three ${ }_{x}(\operatorname{student}(x))$ ". So the interpretation of "three $($ student $(x))$ " does not exclude the possibility that there are more than three students total in the model. But this certainly does not imply that "three $x(\operatorname{student}(x))$ " is identical in meaning to "at_least_three ${ }_{x}($ student $(x))$ ", for only "three $($ student $(x))$ " has as output an information state in which three and only three individuals are assigned to $x$.

(iii) we now proceed to the interpretation of $j^{D i s}(x)$. Since the distributive interpretation is the default in the input information state and has not yet been changed, this test formula doesn't change the input at all. $S_{F}^{2}$ will then pass $j^{D i s}(x)$ and will yield as output $S_{F}^{3}=S_{F}^{2}$.

(iv) Now $S_{F}^{3}$ hits the simple test "Work $(x)$ ". If every individual object that assignment functions in $S_{F}^{3}$ assign to $x$ something that works tirelessly, then $S_{F}^{3}$ will pass the test and output $S_{F}^{4}$.

(v) Now $S_{F}^{4}$ serves as input to the collective transition formula $j^{C o l}(x)$, which the predicate "mowed the whole meadow" requires. In order to pass the transition formula $j^{C o l}(x), S_{F}^{4}$ will change to $S_{F}^{5}$ in which for each $g \in S^{5} \mu^{2}\left(f_{g}(x)\right)$ will be all the objects assigned to $x$ by assignment functions in $S^{4}$. This now serves as the input to the formula " $m o w(x)$ ". Since $S_{F}^{5}$ is the output from $j^{C o l}(x)$, the whether $S_{F}^{5}$ passes " $\operatorname{mow}(x)$ " depends on whether the set of object assigned to $x$ by the assignment functions in $S_{F}^{5}$ belong to the interpretation of mow. If it does, then $S_{F}^{5}$ passes and the output $S_{F}^{6}=S_{F}^{5}$. If not, there is no output from the interpretation of this formula for input $S_{F}^{5}$.

(vi) Now $S_{F}^{6}$ hits the transition formula $j^{D i s}(x)$ which the predicate "had a good time" requires for its interpretation. The output of the transition $j^{D i s}(x)$ is $S_{F}^{7}$, which resets $\mu^{2}\left(f_{g}(x)\right)$ to be just the individual assignments of each $g \in S^{6}$, which is what is needed to properly interpret the distributive predicate "had_good_time $(x)$ ". The output of the interpretation of this last predicate is now available for further discourse interpretation.

To show how our approach treats singular pronouns, we contrast (11) with (12) by looking at their respective logical forms $\left(11^{\prime}\right)$ and $\left(12^{\prime}\right)$ (here the presuppositions and side effects as we have stated them have been added ignoring the other aspects of discourse structure).

(11) Most students go to school. *He brings a lunch box.

(12) Most students go to school. They bring lunch boxes.

(11') $\exists x ; S(x) ; \operatorname{MOSTy}(S(y) ; G(y)) ;$ all $_{x}(G(x)) ;^{*} \operatorname{singular}(x) ; B(x)$

$\left(12^{\prime}\right) \exists x ; S(x) ; \operatorname{MOSTy}(S(y) ; G(y)) ; \operatorname{all}_{x}(G(x)) ; B(x)$

$\left(12^{\prime}\right)$ entails that there is a set of most students that go to school and that all of these students bring lunch boxes. The nature of the witness set for the pronoun is entirely determined by the presupposition and side effects conditions of the determiner. Both $\left(11^{\prime}\right)$ and $\left(12^{\prime}\right)$ have simple distributive readings, which come about because we have 
not reset the default, initial distributive structure of the information state. So there is no problem about interpreting the predicates. But the test $\operatorname{singular}(x)$ checks to see that there is only one value of $x$ per input assignment. Since this is not guaranteed (unless we know a lot more about the context), the logical form $\left(11^{\prime}\right)$ crashes. To guarantee a crash we could stipulate that the plural expression "most students" licenses a set of witnesses that is strictly larger than one as part of the side effects of the determiner.

Let's now consider how dependency affects the use of the singular pronoun. Recall (4) and its logical form with the dependent reading.

(4) Three students wrote a paper. They sent it / them to L\&P.

(4') $\exists x ; \exists y ; \operatorname{three}_{x}(\operatorname{student}(x)) ; j^{D e p}(x, y) ; a_{y}(\operatorname{paper}(y)) ;$ write $(x, y)$; $\operatorname{singular}(y) ; \operatorname{send}(x, y)$

The DPL ${ }^{+}$transition formula " $j{ }^{D e p}(x, y)$ " together with the counting predicate $a_{y}(\operatorname{paper}(y))$ play an important role in getting the right interpretation of "it" in (4). The transition formula partitions up the set of assignments pairing for each given student all the papers associated with him, setting $\mu^{1}\left(f_{g}(y)\right)$ to all the assignments $g^{\prime}$ that agree on a value for $x$, while $a_{y}(\operatorname{paper}(y))$ ensures that the number of papers determined by those assignments in $\mu^{1}\left(f_{g}(y)\right)$ is 1 . This partition persists through the interpretation of the formula write $(x, y)$ and also passes the test given by the interpretation of formula singular $(y)$, since $\mu^{1}\left(f_{g}(y)\right)$ is 1 for every $g$ in the information state.

What happens then with (4) when them is used? them is less picky than it; so the logical form for (4) with the plural pronoun variant would be just $\left(4^{\prime}\right)$ without the formula $\operatorname{singular}(y)$.

This is not the only possible logical form for the discourse. We can replace " $j^{D e p}(x, y)$ " with any other transition formula to get a satisfiable formula; this is not the case with the singular variant-it only has the dependent reading. In fact this would be the same reading as given by (13a) or (13b), where each forces the dependent reading. ${ }^{13}$ Our treatment also easily accounts for the unacceptability of the singular pronoun version of $(13 \mathrm{c})$, since the singular pronoun's requirement will clash with the fact that each student wrote two papers.

(13) a. Three students wrote a paper. They each sent it to L\&P.

b. Three students each wrote a paper. They each sent it to L\&P.

c. Three students each wrote two papers. They sent *it (them) to L\&P.

\subsection{Dependency and Scope}

A rather surprising byproduct of our account of plural readings is that it also captures quantifier readings dependent on scope without the need for any explicit rescoping of quantifiers. Traditional approaches to quantification take the order of the quantifiers to determine various readings of dependency between singular (and plural) quantifiers. So to get different readings let's say of two quantifiers, we need to reorder them on the traditional view. Simple reorderings of plural quantifiers, however, couldn't possibly capture all the different plural readings, and in fact our way of encoding dependency allows us to capture the effects of ordering quantifiers in standard first order logic. We do not need the mechanism of scope to represent different readings. Consider the sentence 
(14) Three students visited two professors.

Two normal scope distributive readings are

- $\exists x ; \exists y ;$ three $_{x}\left(\operatorname{student}_{(x))}\right)$ two $_{y}(\operatorname{professor}(y)) ; \operatorname{visit}(x, y)$, and

- $\exists x ; \exists y ;$ three $_{x}(\operatorname{student}(x)) ; j^{D e p}(x, y) ;$ two $_{y}($ professor $(y)) ; \operatorname{visit}(x, y)$.

To get the so called inverse scope reading of the sentence, we only need the following logic form which does not use scope of quantifiers to account for inverse scope quantifier reading but making use of dependency relations to interpret the inverse quantifier reading: $\exists x ; \exists y ;$ three $_{x}\left(\right.$ student $(x) ;$ two $\left._{y}(\operatorname{professor}(y)) ; j^{D e p}(y, x) ; \operatorname{visit}(x, y)\right){ }^{14}$

The more hackneyed every man loves a woman has two familiar readings that we represent in $\mathrm{DPL}^{+}$as follows (once again presuppositions and side effects have all been integrated into the logical form):

$$
\begin{array}{ll}
\text { a. } & (\exists \forall) \exists x ; \exists y ; M(x) ; \neg \exists x\left(M x ; \neg\left(a_{y}(W(y) ; L x y)\right) ; \text { all }_{x}(M(x)) ;\right. \\
& j^{D i s}(x) ; a_{y}(W(y) ; L x y \\
\text { b. } & (\forall \exists) \exists x ; \exists y ;(M(x)) ; \neg \exists x\left(M x ; \neg\left(a_{y}(W(y) ; L x y)\right) ; \text { all }_{x}(M(x)) ;\right. \\
& j^{D e p}(x, y) ; a_{y}(W(y)) ; L x y^{15}
\end{array}
$$

$\mathrm{DPL}^{+}$codes up the existence of a skolem function where the values for $y$ are dependent on the choice of values for $x$, by using the transition formula $j^{D e p}(x, y) \cdot j^{D i s}(x)$, on the other hand, simply preserves the left right order of the quantifiers in the structure of assignments. Thus, in $\mathrm{DPL}^{+}$we can represent the quantifier readings represented by different scopings of the quantifiers without any movement at the syntactic level, as a byproduct of our analysis plural readings.

\section{Integrating a Richer Notion of Discourse Structure into Our Account}

We've seen that our way of constructing logical forms for English plural discourses in $\mathrm{DPL}^{+}$yields an explanation of some phenomena concerning plural anaphora, without destroying the core dynamic semantic idea that DPs with determiners other than the, one and $a$ can't be the antecedents of singular pronouns. Our dependency transitions allow us access to indefinites under the scope of quantifiers that are normally barriers to anaphoric accessibility in dynamic semantics, but this access requires an explicit relational dependence between the (dependent) anaphor linking to the indefinite and an anaphor to the other quantifier. We also noted that such anaphoric possibilities are rather short lived as in (7) repeated here.

(7) Three students each wrote a paper. They sent it to L\&P. ?They had worked very hard on it.

One possible way to account for this is to stipulate that dependency effects get overridden after the next sentence is processed. But one would like a deeper explanation, presumably one that would also explain the variability of judgements with some of these examples with singular pronouns and antecedents within the scope of a plural existential quantifier. We have a feeling that discourse structure ought to tell us something about these cases, but we don't at the moment know how that story goes.

A further need for discourse structure arises with cases in which a singular pronoun accesses what would normally be an inaccessible antecedent but where no explicit relational dependence between anaphors occurs. 
(16) a. Every frog that saw an insect ate it. It disappeared forever.

b. Each chess set I bought came with a spare pawn. It came taped to the box.

(16a,b) are examples of quantificational subordination, and our framework does not cover these. ${ }^{16}$ We think that these examples in fact would receive a satisfactory treatment in a framework like SDRT where what goes into the nuclear scope of the quantifiers in the first sentences of $(16 a, b)$ might be subject to discourse effects. Certain discourses where the second sentence gives us a result (16a) or an elaboration(16b) allow us to construct logical forms where the nuclear scopes of the quantifiers include in effect material from the second sentence. ${ }^{17}$

SDRT has a theory of how new material gets attached to an antecedently built up discourse structure, whereas our DPL ${ }^{+}$logical forms don't. So SDRT may be able to explain some cases of anaphoric reference that $\mathrm{DPL}^{+}$can't, since in our $\mathrm{DPL}^{+}$ logical forms we've simply appended new material to the logical form for the previous discourse.

However, the details of the SDRT story of these anaphoric cases goes, ${ }^{18}$ we'll need SDRT in any case to build the logical forms that $\mathrm{DPL}^{+}$will interpret. First, we need a theory that integrates presupposed and asserted content and allows us to state side effects of that integration. Further we need a theory in which various information sources like lexical semantics can specify underspecified transitions and can compete and override defaults concerning transition formulas. Finally we need a theory that can account for parallelism effects that motivated us in the first place to think of differences in plural readings as an ambiguity in logical form. SDRT supplies us with all of these things, and it offers us a rich framework within which to explore degradation effects. A fuller discourse based account, however, must wait for another time.

\section{Concluding Remarks}

Many consequences that require further work follow from the semantic framework of $\mathrm{DPL}^{+}$. For one thing we need to look at how conditionals license witness sets for plural anaphora. We also need to address the issue of downward entailing quantifiers like few children, at most five cats, etc. But the framework already shows us how to distinguish various readings of sentences containing plurals that naturally captures the carry over effects that elude many approaches while also handling problematic coordinate VP examples like those in (3). Our framework also gives us a natural way of distinguishing between distributive and dependent readings, a distinction which is implicit in English but explicit in other languages like Hungarian. For us, many plural determiners do introduce ambiguity or underspecification, not in the noun phrase itself, but rather in the transitions that are part of their asserted or side effect conditions. And while we agree to a certain extent with term ambiguity approaches, we àlso think that predicate approaches are right in saying that predicates may force certain plural readings; for us this means that predicates may require certain inputs and thus particular types of dynamic transitions to their left. But once again plural readings can't be simply a matter of how the predicates are interpreted; we couldn't explain the carry over effects in discourse of these readings. For this as well as a unified account of plural readings, we need to adopt a sophisticated dynamic semantics like DPL ${ }^{+}$. 


\section{Endnotes}

${ }^{1}$ To convince yourself that there's a genuine ambiguity here consider Lakoff's (1970) test for ambiguity which states that ambiguities give rise to differences in logical form that are subject to parallelism effects like those brought about by VP ellipsis:

(i) Three students wrote a paper. Four professors wrote a paper too.

This discourses has only two natural readings. The two sentences in (i) either both receive a collective interpretation, or both receive a distributive interpretation. The "mixed" interpretations where the first sentence gets a distributive or collective interpretation and the second receives a different interpretation are difficult to get. Assuming ellipsis is interpreted or reconstructed at logical form and that ambiguities appear as differences in logical form would lead to an explanation of this observation. The situation is quite different where we merely leave some details unspecified, as in John took a trip. Sam took a trip too. This discourse simply leaves the nature of John's trip unspecified; and despite the presence of the parallelism marker too any further specification of the target need not mirror a corresponding specification of the source.

${ }^{2}$ For similar examples and other arguments see also Lasersohn (1995), Link (1993), Roberts (1987), Cameron (1999), and Oliver (2001).

${ }^{3}$ Similar to an example in Cameron (1999).

${ }^{4}$ Our persistence argument affects both DRT style (Kamp and Reyle 1993) and Etype (e.g. Heim 1990) approaches to pronouns. In particular, to see that any term ambiguity approach that appeals to E-type pronouns simply to save itself from the problems we've just mentioned fails, let's consider for a moment how an E-type approach would represent (3b):

(3c) Three students mowed the whole meadow. The three students who mowed the whole meadow worked tirelessly.

Because of the predication in the relative clause, the definite description the second sentence of (3c) must denote a collectively interpreted noun phrase-thus ensuring that the previous reading persists.

${ }^{5}$ See Parson (1970), Zwicky \& Sadock (1975), Margalit (1983), and Gillon (1987), Cruse (1986), and Lascarides, Copestake \& Briscoe (1996).

${ }^{6}$ If the cumulative interpretation cannot be incorporated into the term-ambiguity approach, it is hard for them to provide a consistent picture that different plural readings follow from an ambiguity of plural terms.

${ }^{7}$ This resembles a weakest interpretation approach to this sort of plural reading, something that Kempson \& Cormack (1981) and Verkuyl \& van der Does (1991) have also favored. A weakest interpretation approach is one in which different readings of a sentence are implemented by a single interpretation which is entailed by those different readings of the sentence.

${ }^{8}$ This criticism also applies to an approach that seeks to capture the difference between collective and distributive readings by introducing covert distributivity operators; to handle (3a) the distributivity operator must have scope only over the first VP, but to handle (4) the distributivity operator must have unbound rightward scope.

${ }^{9} \mathrm{E}$-type approaches or some kind of choice function mechanism may be able to account for the interpretation of the singular pronoun in $(5 \mathrm{a}, \mathrm{b})$ by making a functional relation between students and papers. However, it is hard for them to get (4). 
${ }^{10}$ Although our framework can readily accommodate determiners that are monotone decreasing in their second argument like few, at most $n$, etc., we skip the delicate issue of how to treat them.

${ }^{11}$ For details see Asher \& Lascarides (2003).

${ }^{12} \mathrm{DPL}^{+}$has considerably more expressive power than DPL or first order logic (though it does not have the expressive power of full second order logic since we don't quantify directly over arbitrary sets of objects in the domain).

${ }^{13} \mathrm{Krifka}$ (1996) deal with these cases in a different way.

${ }^{14}$ We can put the logic form in the following more general way: $\exists x ; \exists y ; j^{D e p}(y, x)$; three $_{x}\left(\operatorname{student}_{(x))}\right)$ two $_{y}($ professor $(y)) ; \operatorname{visit}(x, y)$

${ }^{15}$ Or the following logic form: $\exists x ; \exists y ; M(x) ; \neg \exists x\left(M x ; \neg\left(a_{y}(W(y) ; L x y)\right)\right.$; $j^{D e p}(x, y) ; \operatorname{all}_{x}(M(x)) ; a_{y}(W(y)) ; L x y$

${ }^{16}$ Gawron (1996) also cannot account for these examples, even though he can handle some cases of quantificational subordination-viz., those in which there is another quantificational element in the second sentence like an adverb of quantification.

${ }^{17}$ This is in effect similar to Sells's (1985) insertion approach to modal and quantificational subordination, though in Sells's work there is no means for studying the discourse effects that license this incorporation.

${ }^{18}$ See Asher (2001) for more details.

\section{References}

Asher, N. (1993), Reference to Abstract Objects in Discourse, Kluwer Academic Publishers.

Asher, N. (2001), 'Plurals in Discourse', manuscript.

Asher, N., \& Lascarides, A. (2003), Logic of Conversation, Cambridge University Press.

Boolos, G. (1984), "To Be is to Be a Value of a Variable (Or to Be Some Values of Some Variables)," Journal of Philosophy, 81, pp. 430-448.

Cameron, R. (1999), "Plural Reference," Ratio, XII(2), pp. 128-147.

Cruse, D. (1986), Lexical Semantics, Cambridge: Cambridge University Press.

Farkas, D. (1997), "Dependent Indefinites," in F. Corblin \& D. Godard \& J.-M. Marandin (1997), (eds.), Empirical Issues in Formal Syntax and Semantics, Peter Lang Publishers, pp. 243-268.

Gawron, M. (1996), "Quantification, Quantificational Domains, and Dynamic Logic," in S. Lappin (1996), (ed.), The Handbook of Contemporary Semantic Theory, MA: Blackwell Publishers, pp. 247-267.

Gillon, B. (1987), "The Readings of Plural Noun Phrases in English," Linguistics and Philosophy, 10, pp. 199-219.

Groenendijk, J. \& Stokhof, M. (1991), “Dynamic Predicate Logic," Linguistics and Philosophy, pp. 39-100.

Heim, I. (1982), The Semantics of Definite and Indefinite Noun Phrase in English, Dissertation, University of Massachusetts at Amherst.

Heim, I. (1990), "E-Type Pronouns and Donkey Anaphora," Linguistics and Philosophy, 13, pp. 137-177.

Kamp, H. (1981), "A Theory of Truth and Semantic Representation," reprinted in Groenendijk, J., Janssen, T. \& Stokhof, M. (1984), (eds.), Truth, Representation, and Information, Dordrecht: Foris, pp. 277-322. 
Kamp, H. and Reyle, U. (1993), From Discourse to Logic: Introduction to Model Theoretic Semantics of Natural Language, Formal Logic and Discourse Representation Theory, Dordrecht; Boston: Kluwer Academic.

Kempson, R. \& Cormack, A. (1981), "Ambiguity and Quantification," Linguistics and Philosophy, 4, pp. 259-309.

Krifka, M. (1996), "Parametrized Sum Individuals for Plural Anaphora,"Linguistics and Philosophy, pp. 555-598.

Lakoff, G. (1970), "A Note on Vagueness and Ambiguity," Linguists Inquiry, 1(3), pp. 357-359.

Landman, F. (2000), Events and Plurality: the Jerusalem Lectures, Dordrecht: Kluwer Academic Publishers.

Lascarides, A., Copestake, A., \& Briscoe, T. (1996), "Ambiguity and Coherence," Journal of Semantics, 13(1), pp. 41-65.

Lasersohn, P. (1995), Plurality, Conjunction and Events, Dordrecht: Kluwer Academic Publishers.

Link, G. (1993), "Plural," in D. Wunderlich \& A. von Stechow, (1993), (eds.), Handbook of Semantics, Berlin: de Gruyter.

Oliver, A., \& Smiley, T. (2001), "Strategies for A Logic of Plurals," The Philosophical Quarterly, 51, pp. 289-306.

Parsons, K. P. (1973), "Ambiguity and the Truth Definition," Nous, 7(4), pp. 379-394.

Roberts, C. (1987), Modal Subordination, Anaphora, and Distributivity, Dissertation, UMass, Amherst.

Scha, R. (1981), "Distributive, Collective, and Cumulative Quantification," in J. Groenendijk, T. Janssen \& M. Stokhof, (1981), (eds.), Formal Methods in the Study of Language, Amsterdam: Mathematical Center Tracts.

Schein, B. (1993), Plurals and Events, Cambridge, Mass.: MIT Press.

Sells, P. (1985), Restrictive and Non-Restrictive Modification, Center for the Study of Language and Information, Report No. CSLI-85-28.

van den Berg, M. (1996), Some Aspects of the Internal Structure of Discourse: The Dynamics of Nominal Anaphora, Dissertation, University of Amsterdam.

van der Sandt, R. (1992), "Presupposition projection as anaphora resolution," Journal of Semantics, 9(2), pp. 333-377.

Verkuyl, H., \& van der Does, J. (1991), "The Semantics of Plural Noun Phrases," in J. van der Does \& J. van Eijck, (1991), (eds.), Quantifiers: Logic and Language, Standford: CSLI Publication, pp. 403-441).

Winter, Y. (2001), Flexibility Principles in Boolean Semantics, Massachusetts: The MIT Press.

Zwichy, A., \& Sadock, J. (1975), "Ambiguity Test and How to Fail Them," in J. Kimball, (1975), (ed.), Syntax and Semantics, 4, pp. 1-36. 